\title{
Geographic Tongue in Psoriasis: Case Reports
}

\author{
Ariyati Yosi, Cashtry Meher, Irma D. Roesyanto-Mahadi \\ Dermatology and Venereology Department \\ Universitas Sumatera Utara/H. Adam Malik General Hospital \\ Medan, Indonesia
}

\begin{abstract}
Geographic tongue is an idiopathic inflammatory disorder resulting in the local loss of filiform papillae, which is present as erythematous patches with serpiginous borders resembling a map. The etiopathogenesis of geographic tongue is unknown. Geographic tongue has been postulated to be an oral variant of psoriasis as this lesion show several histologic features of psoriasis. Two cases of asymptomatic geographic tongue in psoriasis patients are presented here. Geographic tongue has been postulated as oral variant of psoriasis since the prevalent of this condition is increase in psoriatic patient; they have same several histologic features, and also have the same human leukocyte antigen (HLA) group. The prevalence of geographic tongue is increased in psoriastic patients. The conclusion of this case reports was geographic tongue seems to have relationship with psoriasis since they have similar histologic and HLA group.
\end{abstract}

Keywords-geographic tongue, psoriasis, loss of filiform papillae, chronic skin disorder

\section{INTRODUCTION}

Geographic tongue is an idiopathic inflammatory disorder resulting in the local loss of filiform papillae, present as erythematous patches with serpiginous borders resembling a map [1,2]. It is affecting the dorsum and margin of the tongue [2]. The etiopathogenesis of geographic tongue is unknown. Several factors are suggesting have relationship with geographic tongue [2-4]. This condition occurs in $1 \%$ to $3 \%$ of the population, common in young and middle aged adult, and females are affected more frequently than males $[2,3]$.

Geographic tongue has been postulated to be an oral variant of psoriasis, as these lesion show several histologic features of psoriasis, called a psoriasiform mucositis. Psoriasis patients and those with geographic tongue also have the same human leukocyte antigen (HLA) group. Furthermore the prevalence of geographic tongue is increased in psoriasis patients than in the general population, occurred about $10 \%$. However, geographic tongue is a relatively common condition and is seen in many nonpsoriatic individuals, so its relationship to psoriasis needs further clarification $[1,3]$.

Hence the aim of this case reports are therefore to discuss 2 case of psoriasis with geographic tongue. The case have different course of disease and psoriasis index.

\section{CASE REPORT}

\section{A. Case 1}

A 47-year-old man presented for evaluation of his flare up of pre-existing plaque-type psoriasis. The patient developed plaque-type psoriasis since 6 months ago, and he has been experiencing exacerbations within 10 days. He has been treated with high-potency corticosteroids and calcipotriol topically. On physical examination we found unusual tongue feature without any complain from the patient. The patient said he already has this condition since 3 months ago. The initial lesion noted as small white patches that became larger. The vital signs are within normal limits. He had inflammatory psoriatic patches and plaques of his skin. Psoriasis Area and Severity Index (PASI) score for this patient was 28.8. Oral examination revealed welldemarcated erythematous patches on dorsal and lateral distribution of the tongue (Figure 1).

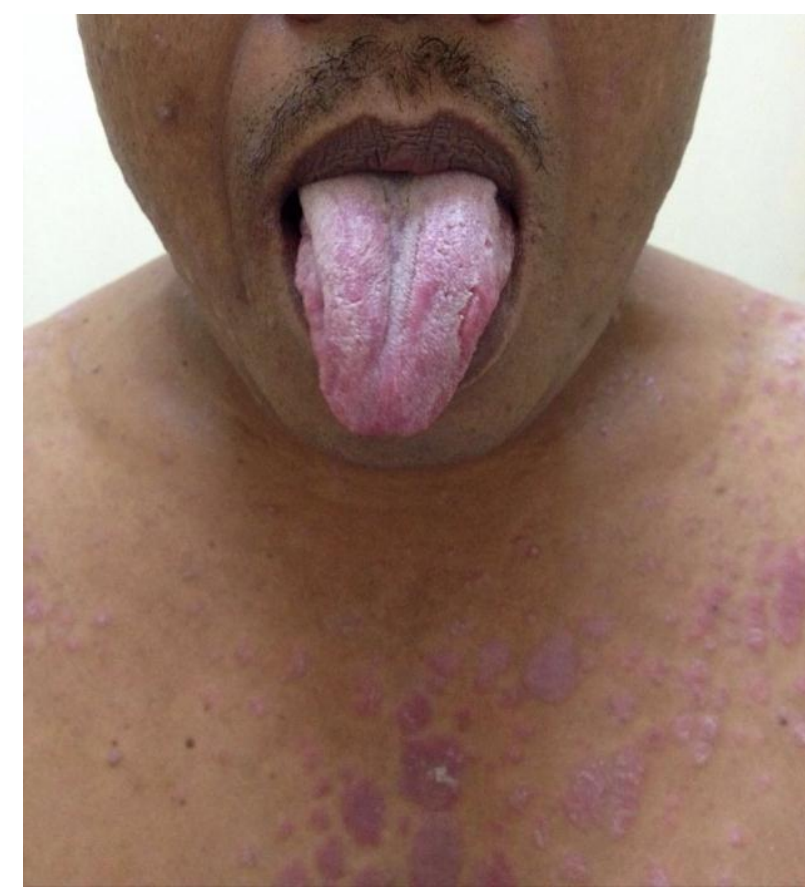

Figure 1. Well-demarcated erythematous patches on dorsal and lateral of the tongue.

\section{B. Case 2}

A 42-year-old man, diagnosed as psoriasis vulgaris, presented for his regular evaluation. The patient suffered psoriasis since 1 year ago. He has been treated with low-potency topical corticosteroids and emollient 
for maintenance therapy. On physical examination we found unusual tongue feature. The patient was unaware of condition and had no pain or discomfort. He realized this condition about 4 months ago when he saw uneven color of his tongue. On physical examination, the vital signs within normal limits. The psoriatic patches seem normal without much scale on the patches. The PASI score for this patient was 10.8. Oral examination revealed well-demarcated erythematous patches on lateral tongue (Figure 2).

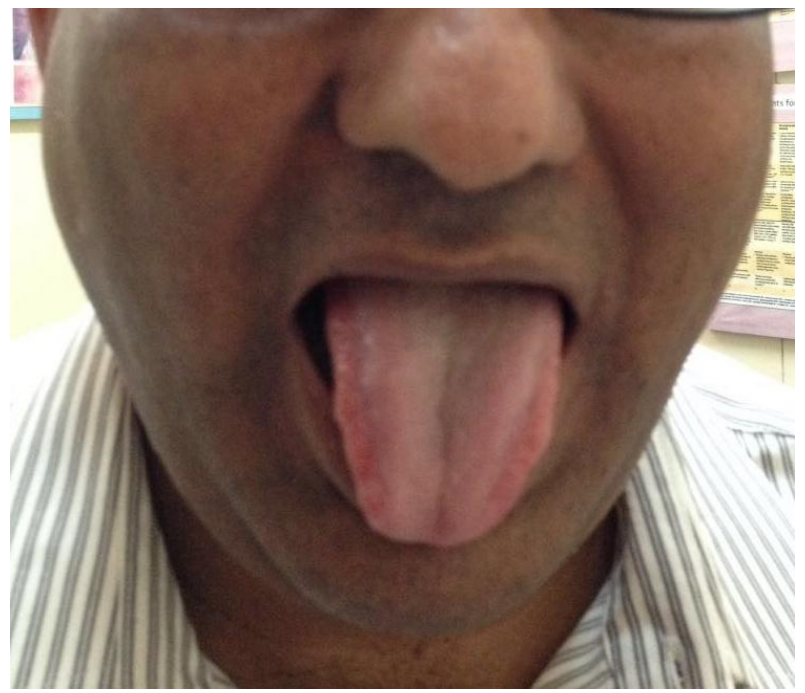

Figure 2. Well-demarcated erythematous patches on lateral tongue.

\section{DISCUSSION}

Geographic tongue, also known as benign migratory glossitis or glossitis areata migrans/exfoliativa or erythema migrans or wandering rash, is one of the most prevalent oral mucosal lesions $[1,2,4]$. The etiopathogenesis of geographic tongue is unknown. Several factors are suggesting have relationship with geographic tongue, including hereditary factor, atopic individuals, cigarette smokers, immunological reaction, emotional stress, infections, and nutritional deficiencies [2-4]. But also many the studies revealed a negative relationship to these factors [2].

Geographic tongue occurs in $1 \%$ to $3 \%$ of the population [3]. The gender distribution appears to be equal, but some literatures reported females are affected more frequently than males, about $2: 1$ ratio [2-4]. This condition common in young and middle aged adult, supports theory of spontaneous regression over time $[2,3]$.

Geographic tongue is circumferentially area leaves an erythematous area behind reflecting of the filiform papillae. The lesion may commence at different starting points, the peripheral zone fuse, and the typical clinical features of a geographic tongue emerge [1,2]. The lesion may vary from single to multiple [2]. The disorder is usually asymptomatic, but some patients are experiencing a sensitive, burning or painful, especially when acidic or spicy foods come in contact with it $[1,2,4,5]$.
The clinical features such demarcated areas rimmed by a raised white border that is circinated or serpiginous are diagnostic for geographic tongue, and histologic confirmation is rarely needed [2,5]. The differential diagnosis of patient with geographic tongue might be erythematous candidiasis. The identification of the candidal hyphae is necessary for this condition [5]. No causal treatment is available for the disease as the etiology is unknown. In symptomatic condition, topical anesthetics may be used. Others suggesting treatment strategies are antihistamines, anxiolytic drugs, and steroid. $^{2}$ If severe, topical steroid (especially flucinonide and dexamethasone) are helpful [3,5]. Geographic tongue may regress within few days or weeks $[2,3]$

Psoriasis is a chronic inflammatory disorder of the skin and articular with polygenic predisposition combined with triggering environmental factor such as trauma, infection and medication [1,6]. Its prevalence in different population varies from $0.1 \%$ to $11.8 \%$. The skin lesion also have different manifestation, including scaly papules and plaques, pustular or erythrodermic eruptions, depends of its type. Most common sites in involvement are scalp, elbow, knees, hands, feet, trunk and nails [1].

Geographic tongue has been postulated as oral variant of psoriasis since the prevalent of this condition is increase in psoriatic patient; they have same several histologic features, and also have the same human leukocyte antigen (HLA) group [1,2]. But, since geographic usually appear without an apparent association to the cutaneous disease, the association still questioned [7].

The prevalence of geographic tongue is increased in psoriastic patients [1]. Approximately $13 \%$ of patient with psoriasis develop this tongue condition, more than it's prevalence in non-psoriasis population [5]. From 207 patients in one study, geographic tongue was present in $12.5 \%$ of the group with psoriasis and $4.7 \%$ in the group without this disease [7]. Other study in 400 psoriasis patients, there was a highly significant $(\mathrm{P}<0.0001)$ correlation between geographic tongue and psoriasis $(7.7 \%$ of psoriasis patients versus $1 \%$ of controls) [8].

One study in India which evaluated 600 psoriatic patients showed that $79 \%$ of psoriatic patients with geographic tongue were male. This data differs from patients with geographic tongue without psoriasis who are usually female [9]. Our report also showed that both our case is male patients. But in other study, there were no gender differences in psoriasis patients with geographic tongue [10].

Type of psoriasis can be made by its clinical pattern of skin presentation. It is including psoriasis vulgaris/chronic stationary psoriasis/plaque type psoriasis, guttate/eruptive psoriasis, small plaque psoriasis, inverse/flexural psoriasis, and erythrodermic psoriasis and pustular psoriasis [1]. Geographic tongue especially seen in generalized pustular psoriasis, ${ }^{\text {but }}$ in a multicentric study with plaque-type psoriasis patients, 
geographic tongue was seen more frequently in psoriatic patients $(9.1 \%)$ than the control group $(5.2 \%)$ $(p<0.05)[1,2,10]$. Also, Our patients above are plaque type psoriasis patients. The presence of geographic tongue in a normal patient indicates an increased likelihood of developing generalized pustular psoriasis [11].

In a study, Geographic tongue was seen in $7.2 \%$ of patients with early onset psoriasis and only in $1.3 \%$ of patients with late-onset psoriasis, suggesting this condition as an indicator for severity of psoriasis [12]. In other study, there were no significant differences [7]. Both of our patient are late-onset psoriasis patients since the onset of their psoriasis is starting after the age 47 (case 1) and 41 (case 2). According to age of psoriasis onset, the individuals classified as having early psoriasis (stating before or equal the age of 40) and late psoriasis (after the age of 40)[7]. Also, our patients have different severity of the disease with PASI score 28. 8 and 10.8. PASI (Psoriasis Area and Severity Index) is one of tools used to determined severity of psoriasis that commonly used in clinical trials for psoriasis treatments and by dermatologists and clinicians working in specialized treatment centers. PASI is measure of overall severity and extent of psoriasis by assessing body surface area and intensity of redness, thickness and scaling. A single score is calculated and ranges between 0 (no disease) to 72 (maximal disease). In a study, frequency of geographic tongue increased with the severity of psoriasis in plaque-type psoriasis assessed by PASI score [11]. Moreover, one of our patients came with exacerbated disease (case 1) and other patient is maintenance phase patient (case 2).

The similar histologic features in geographic tongue and psoriasis are including acanthosis, clubbing and elongation of the rete ridges, focal parakeratosis, and subepithelial inflammation of $\mathrm{T}$ lymphocytes. In addition, the transepithelial migrating neutrophilic granulocyte infiltrate can be observed $[1,3]$. These cells may be a part of microabscesses formed near the surface that similar with Munro's microabscesses (neutrophil accumulation in epidermal stratum corneum), and similar with spongiform pustules of Kojog, (neutrophil accumulation in spinous layer of epidermis) in psoriasis lesion, especially in pustular psoriasis type. This histologic finding of neutrophil accumulation was seen in the center area of geographic tongue, but rarely found in peripheral zone of the lesion [1-3]. The intense neutrophilic infiltrate may be responsible for the destruction of the superficial portion of epithelium, thus producing an atrophic, reddened mucosa. Because these similar histopathologic features, this is called a psoriasiform mucositis [3]. Different as geographic tongue, the role of neutrophils in psoriasis pathogenesis is unclear; even in some studies indicate that neutrophils are probably unnecessary for psoriasis lesional development [1].

Psoriasis patients and those with geographic tongue also have the same human leukocyte antigen (HLA) group, namely HLA-Cw6 [1,3]. As has been made clear by detailed fine mapping, genetic linkage, and association studies, HLA-Cw6 is by far the major genetic risk factor for psoriasis [1]. In a study of 22 patients reported a highly significant association of Cw6 with both psoriasis and benign migratory glossitis, with this antigen being present in $59.1 \%$ of the patients of psoriasis, $43.8 \%$ of the patients with benign migratory glossitis and in only $12.6 \%$ of the controls [13].

However, many other HLA group have been known in psoriasis and geographic tongue. In psoriasis, other HLA have relationship with the disease, including HLA-Cw2, Cw4, Cw5, Cw15 and Cw17 [1]. Likewise, an increased incidence of HLA-B15, DR5 and DRW6 antigens was found in geographic tongue patients $[14,15]$.

The conclusion of this case reports was geographic tongue seems to have relationship with psoriasis since they have similar histologic and HLA group. Prevalence of geographic tongue is also higher in psoriasis patients. But, studies should be conducted to know the further relationship between geographic tongue and psoriasis.

\section{ACKNOWLEDGMENT}

The acknowledgments are addressed to The Adam Malik General hospital Medan The author would like to offer the outmost gratitude to this institution for fully supporting and funding of this review.

\section{REFERENCES}

[1] J.E. Gudjonsson, J.T. Elder, Psoriasis. In: Fitzpatrick's dermatology in general medicine, $8^{\text {th }}$ ed., New York: Mc Graw Hill, 2012, pp. 216

[2] M. Jontell, P. Holmstrup, Red and white lesions of the oral mucosa. In: Burket's oral medicine, $12^{\text {th }}$ ed., Connecticut: People's Medical Publishing House, 2015, pp. 118-119

[3] B.W. Neville, D.D. Damm, C.M. Allen, J.E. Bouquot, Oral and maxillofacial pathology, $3^{\text {rd }}$ ed., Singapore: Sauders Elsevier, 2012, pp.779-781

[4] A.G. Ghom, S.A. Ghom, eds. Textbook of oral medicine, $3^{\text {rd }}$ ed., New Delhi: Jaypee Brothers medical publishers, 2014, pp. 512513

[5] S.B. Woo, Biology and pathology of the oral cavity. In: Fitzpatrick's dermatology in general medicine, $8^{\text {th }}$ ed., New York: Mc Graw Hill, 2012, pp. 850

[6] V. Ngan, A. Oakley (2017, November 4) Guideline for the management of psoriasis. Available: https://www.dermnetnz.org/topics/guidelines-for-themanagement-of-psoriasis/

[7] F.I. Hernández-Pùrez, A. Jaimes-Aveldañez, L. UrquizoRuvalcabamde, M. Díaz-Barcelot, M.E. Irigoyen-Camacho, M.E. Vega-Memije, et al, "Prevalence of oral lesions in patients with psoriasis," Med. Oral Patol. Oral Cir. Bucal, vol. 13(11) pp. E703-708, 2008.

[8] R. Tomb, H. Hajj, E. Nehme, "Oral lesions in psoriasis," Ann Dermatol. Venereol., vol. 137(11), pp. 695-702.

[9] S. Singh, S. Nivash, B.K. Mann, "Matched case-control study to examine association of psoriasis and migratory glossitis in India," Indian J. Dermatol. Venereol. Leprol., vol. 79, pp. 5964.

[10] L. Germi L, V. De Giorgi, F. Bergamo, M.C. Niccoli, F. Kokelj, M. Simonacci, et al, (2017, November 6) Psoriasis and oral lesions: Multicentric study of oral mucosa diseases italian group 
(GIPMO), Dermatology Online Journal. Available: https://escholarship.org/uc/item/4610f41r

[11] M. Daneshpazhooh, H. Moslehi, M. Akhyani, M. Etesami, "Tongue lesions in psoriasis: a controlled study," BMC Dermatol., vol. 4, pp. 16-16, 2004.

[12] O. Zargari, "The prevalence and significance of fissured tongue and geographical tongue in psoriatic patients," Clin. Exp. Dermatol., vol. 2, pp. 192-95, 2006.
[13] H.F. Gonzaga, E.A. Torres, M.M. Alchorne, M. GerbaseDelima, "Both psoriasis and benign migratory glossitis are associated with HLA-Cw6," Br. J. Dermatol., vol. 135, pp. 368370, 2006

[14] R. Marks, B. Taitt, "HLA antigens in geographic tongue," Tissue Antigens, vol. 15, pp. 60-62, 1980.

[15] A.I. Fenerli, S. Papanicolaou, M. Papanicolaou, G. Laskaris, "Histocompatibility antigens and geographic tongue," Oral Surg. Oral Med. Oral Pathol., vol. 76, pp. 476-479, 1993. 